Received Date : 13-Apr-2016

Revised Date : 28-Jul-2016

Accepted Date : $16-$ Sep-2016

Article type : Original Papers

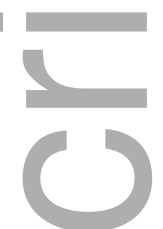

\title{
Cerebral small vessel disease, medial temporal lobe atrophy and cognitive status in patients with ischaemic stroke and transient ischaemic attack
}

Francesco Arba, MD, $\mathrm{PhD}^{1,4}$; Terence Quinn, $\mathrm{MD}^{2}$; Graeme J. Hankey MD ${ }^{3}$; Myzoon Ali, $\mathrm{PhD}^{4}$; Kennedy R. Lees MD, $\mathrm{FRCP}^{5}$; Domenico Inzitari, $\mathrm{MD}^{1}$; On behalf of the VISTA Collaboration*

1- NEUROFARBA Department, University of Florence, Florence, Italy

2- Institute of Cardiovascular and Medical Sciences, University of Glasgow, Glasgow Royal Infirmary, Glasgow, UK

3- School of Medicine and Pharmacology, University of Western Australia, Harry Perkins Institute of Medical Research, QEII Medical Centre, Perth, Australia

4- Institute of Cardiovascular and Medical Sciences, Queen Elizabeth University Hospital Glasgow, Glasgow, UK

5- Institute of Cardiovascular \& Medical Sciences, University of Glasgow, C249 BHF Building, Glasgow, UK

Corresponding author: Francesco Arba

This is the author manuscript accepted for publication and has undergone full peer review but has not been through the copyediting, typesetting, pagination and proofreading process, which may lead to differences between this version and the Version of Record. Please cite this article as doi: 10.1111/ene.13191

This article is protected by copyright. All rights reserved 
Fax: +390557947665

Phone: +39055 7947955

Email: francesco.arba@unifi.it

Running title: small vessel disease, neurodegeneration and cognitive status after stroke

Keywords: stroke, small vessel disease, cognitive disorders and dementia, medial temporal lobe atrophy, Alzheimer Disease

\section{Abstract}

Background: Small vessel disease (SVD) and Alzheimer disease (AD) are two common causes of cognitive impairment and dementia, traditionally considered as distinct processes. We explored the relationship between radiological features suggestive of AD and SVD, and investigated the association of each of these features with cognitive status at one year in patients with stroke or transient ischaemic attack.

Methods: Anonymised data were accessed from the Virtual International Stroke Trials Archive (VISTA). We rated medial temporal lobe atrophy (MTA; a marker of AD) and markers of SVD using validated ordinal visual scales. Cognitive status was evaluated with Mini Mental State Examination (MMSE) one year after the index stroke. We used logistic regression models to investigate independent associations between: 1-baseline SVD features and MTA, and 2-all baseline neuroimaging features and cognitive status one year post-stroke.

Results: We included 234 patients, mean $( \pm$ SD) age=65.7 \pm 13.1 years, $145(62 \%)$ male. Moderate to severe MTA was present in 104 (44\%) patients. SVD features were independently associated with MTA $(\mathrm{p}<0.001)$. After adjusting for age, sex, disability after stroke, hypertension and diabetes mellitus, MTA was the only radiological feature independently associated with cognitive impairment, defined using thresholds of MMSE $\leq 26$ $(\mathrm{OR}=1.94 ; 95 \% \mathrm{CI}=1.28-2.94)$ and $\mathrm{MMSE} \leq 23(\mathrm{OR}=2.31 ; 95 \% \mathrm{CI}=1.48-3.62)$.

Conclusion: In patients with ischaemic cerebrovascular disease, SVD features are associated with MTA, which is a common finding in stroke survivors. SVD and AD-type neurodegeneration co-exist, but the AD marker MTA, rather than SVD markers, is associated with post stroke cognitive impairment.

\section{Introduction}


Dementia is a major public health issue, affecting approximately 47 million people worldwide, by 2030, this figure will increase to approximately 75 million ${ }^{1}$. After Alzheimer disease $(\mathrm{AD})$, vascular dementia $(\mathrm{VaD})$ is the second most common type of dementia. The pathological processes that contribute to $\mathrm{AD}$ and $\mathrm{VaD}$ differ, with primary neuronal degeneration considered to be the pathological process that contributes to $\mathrm{AD}$, and cerebral small vessel disease (SVD) a major contributor to $\mathrm{VaD}$. Medial temporal lobe atrophy (MTA) is a major neurodegenerative dementia marker that is traditionally associated with the clinical diagnosis of $\mathrm{AD}^{2}$. In contrast, the radiological features of SVD encompass a wide range of imaging phenotypes, ranging from white matter changes (WMCs), to lacunar infarcts and enlarged perivascular spaces (EPVS) ${ }^{3}$.

AD and SVD are usually considered distinct entities with differing neuroradiological appearances. The concept of exclusive categorizations of AD and vascular dementia (VaD) has been challenged. A recent scientific statement underscored the importance of the vascular contribution to all cause cognitive impairment and dementia ${ }^{4}$, while observational data suggest that vascular risk factors such as hypertension and diabetes may play an important role in pathogenesis and progression of $\mathrm{AD}^{5,6,7}$.

Following stroke, a third of patients develop dementia, and up to two thirds have some degree of persisting cognitive impairment ${ }^{8}$. Establishing the pathogenesis of post stroke cognitive impairment may allow for development of targeted therapy. The impaired cognition that follows stroke is traditionally considered to be of vascular origin, however it seems plausible that a substantial proportion with post stroke cognitive impairment may have co-existing AD type pathology. In this regard, the evidence about vascular and neurodegenerative contribution to cognitive impairment after stroke is not definitive, and the overlap between such contributors remains overlooked ${ }^{9}$. Therefore, our aim was to evaluate the relationship between neuroimaging markers of $\mathrm{AD}, \mathrm{SVD}$ and cognition in a cohort of patients with ischaemic stroke, extending the existing evidence.

Our specific objectives were to:

a) describe the association between SVD and an exemplar AD neuroimaging marker (MTA)

b) describe the association between these neuroimaging features and cognitive status one year after the index stroke. 


\section{Methods}

\section{Participants}

We conducted individual patient level data analyses using the Virtual International Stroke Trial Archive (VISTA). VISTA is an academic collaboration that provides access to existing anonymised trial data to perform exploratory analyses; to allow hypothesis testing and to inform the design of future clinical trials ${ }^{10}$.

From the VISTA resource, we included those patients with diagnosis of ischaemic stroke and transient ischaemic attack (TIA), where magnetic resonance (MR) imaging of brain and evaluation of cognitive status (e.g., cognitive scales, clinical evaluation) were available. All included trials required valid consent. Clinical variables of interest included: age, sex, clinical history of hypertension, diabetes mellitus, atrial fibrillation, hypercholesterolemia, peripheral artery disease, ischaemic heart disease, cigarette smoke exposure and depression (all defined as per parent trials). Included participants had vascular, laboratory and cardiac investigation data available; characterisation of stroke aetiology was assessed according to the method described in the Trial of Org 10172 (TOAST) ${ }^{11}$. Function was assessed at baseline (i.e. after the index stroke) and quantified using the Oxford Handicap Scale (OHS) ${ }^{12}$; and used as a proxy for stroke severity. OHS is a derivative of the modified Rankin Scale, which is the most common outcome measure in stroke trials.

\section{Imaging analysis}

A stroke neurologist trained in MR assessment and blinded to clinical data (FA) rated all the available scans (T1, T2, FLAIR sequences). SVD features were rated according to STRIVE (STandards for ReportIng Vascular changes on nEuroimaging) recommendations ${ }^{13}$. Where the index infarct was too large to allow the rating of the SVD features, we performed SVD ratings only in the non-affected hemisphere. We defined lacunes as round shaped cerebrospinal fluid isointense lesions measuring $\leq 20 \mathrm{~mm}$ in diameter on axial section in the white matter, basal ganglia or brainstem as seen on T1, T2 or FLAIR sequences. We graded white matter hyperintensities according to Van Swieten scale ${ }^{14}$ as 0-4, combining the ratings in the anterior and posterior periventricular white matter. Brain atrophy was defined as central and cortical, and rated as none, moderate and severe against a reference MR brain template $^{15}$. Enlarged perivascular spaces (EPVS) were defined as $\leq 2 \mathrm{~mm}$ round or linear 
cerebrospinal fluid isointense lesions (T2 hyperintense and T1/FLAIR hypointense). We separately rated EPVS in basal ganglia and centrum semiovale using a 5-point ordinal scale ${ }^{16}$ as follows: $0=$ no EPVS, 1=1-10 EPVS, $2=11$ to 20, EPVS, 3=21 to 40 EPVS, and 4= $>40$ EPVS. As a marker of neurodegeneration classically seen in AD, we rated MTA with coronal T1 weighted sequences in 0-4 with a validated visual scale ${ }^{2}$ and against a reference template.

\section{Cognitive status}

We included studies with cognitive assessment after one year the index stroke. Cognitive impairment was assessed using the Mini Mental State Examination (MMSE) ${ }^{17}$. We defined cognitive impairment as MMSE $\leq 26^{18}$. We used an alternative threshold, said to be more specific to dementia MMSE $\leq 23$. We excluded patients with a baseline diagnosis of dementia from our analysis $(n=6)$.

\section{Statistical analysis}

After taking into account the distribution of data, we used Spearman's rank correlation coefficient to evaluate the relationship between each of the SVD MR features and MTA. We described MMSE scores, stratified by MTA grading and analyzed between-group differences with Kruskal-Wallis test. To assess multivariable association with MTA, we used logistic regression. We dichotomized MTA into groups of: 0-1 (none to mild MTA) vs 2-4 (moderate to severe). We described univariate associations between SVD features and dichotomized MTA. For our multivariable models, we retained statistically significant $(\mathrm{p}<0.1)$ variables from the univariate analysis. We also included variables with face validity or where previous work has suggested an association with cognition, such as age, sex, history of hypertension and history of diabetes.

We described univariate associations between all neuroimaging features (both SVD and MTA, considered as ordinal scales) and cognitive status. We similarly created a multivariate model comprising significant and known associated variables as described previously. For multivariate models we described adjusted odds-ratios (OR) and their $95 \%$ confidence intervals (CI); statistical significance was defined as $\mathrm{p}<0.05$.

\section{Results}

A total of 234 patients had full clinical, radiological and cognitive data. Mean $( \pm \mathrm{SD})$ age was $65.7( \pm 13.1), 145$ patients $(62 \%)$ were male. One hundred and ninety five patients had 
ischaemic stroke as index event (83\%), of whom 94 (40\%) had lacunar stroke subtype (Table $1)$.

One hundred and four (44\%) patients were assessed to have moderate to severe MTA (a score of 2 to 4). Compared to patients with no to mild MTA, those with moderate to severe MTA were older $(60.2$ vs 70.5 years, $\mathrm{p}<0.001)$, more frequently had a history of hypertension $(73 \%$ vs $87 \%, \mathrm{p}<0.012$ ), had a higher grade of disability at baseline (median $\mathrm{OHS}=1$ vs $2, \mathrm{p}<0.001$ ) and more frequently had evidence of our chosen SVD neuroimaging features (all $\mathrm{P}<0.001)$. (Table 1)

MTA had positive correlations with all SVD features. MTA was most closely related with central brain atrophy $(\rho=0.64, p<0.001)$ and white matter changes $(\rho=0.60, p<0.001)$, the weakest correlations were with number of lacunes $(\rho=0.32, p<0.001)$ and centrum semiovale EPVS $(\rho=0.35, p<0.001)$. (Table 2)

In logistic regression models, after adjusting for age, sex, hypertension and diabetes mellitus, several radiological features of SVD remained independently associated with MTA (Table 3). Only the association between centrum semiovale EPVS and MTA failed to reach statistical significance in our sample $(\mathrm{OR}=1.47 ; 95 \% \mathrm{CI}=0.99-2.18, \mathrm{p}=0.057)$, even though the magnitude of effect was similar to the unadjusted analysis.

One year after index stroke, 101 (43\%) patients had cognitive impairment as defined by MMSE $\leq 26$, whereas 67 (29\%) had MMSE $\leq 23$, suggestive of dementia. Distribution of MMSE scores among MTA grades showed a decline in cognitive performance with increasing severity of MTA ( $<<0.001$, Figure 1). In the univariable logistic regression models, all the radiological variables were associated with development of cognitive impairment at one year, and only lacunes were not significantly associated with dementia. However, after adjustment for age, sex, disability status, hypertension and diabetes mellitus, only MTA remained independently associated with $\mathrm{MMSE} \leq 26(\mathrm{OR}=1.94$ per point increase in MTA score; 95\% $\mathrm{CI}=1.28-2.94)$ and with $\mathrm{MMSE} \leq 23(\mathrm{OR}=2.31 ; 95 \% \mathrm{CI}=1.48-3.62)$.

\section{Discussion}


We found that MTA is a common finding in clinical stroke trial population, and has important implications for cognition status at 1 year post-stroke. MTA and SVD may coexist, but MTA seems to have a stronger association with cognitive status than SVD markers.

To date, few studies have specifically addressed the potential overlap between radiological markers of SVD and neurodegeneration markers in a population of patients with ischaemic stroke. In our clinical trial based population, MTA was present in $40 \%$ of patients, a proportion comparable with previous studies ${ }^{19,20,21}$. We found that MTA strongly correlated with brain features that may be seen in any dementia process, for example atrophy. However, we also demonstrated correlation of MTA with neuroimaging features usually described as exclusively relating to small vessel cerebrovascular disease, for example lacunes and basal ganglia EPVS. The independent association between SVD radiological markers and MTA alludes to a potential overlap between neurodegenerative and neurovascular pathology. Other groups have also reported this shared pathology, with WMCs described in patients with $\mathrm{AD}^{22}$ and MTA present in more than half of elderly stroke survivors ${ }^{20,21}$ and in patients with diagnosis of vascular dementia ${ }^{23}$. Indeed, many groups would say that most post-stroke cognitive impairment can be predominantly classified as vascular dementia.

In our study, MTA severity was independently associated with cognitive impairment and dementia. It is perhaps unsurprising that small vessel cerebrovascular features have limited association with cognition after correction for vascular risk factors, as SVD is so closely related to hypertension and diabetes mellitus. Our results support that SVD and AD frequently co-exist but are not synonymous, as MTA still demonstrated cognitive association even after correction for vascular risk factors. Our results are in line with a previous study that showed MTA rather than white matter changes as the strongest marker of long-term memory function after stroke ${ }^{24}$.

However, we did not have pre-stroke assessment of cognitive status. It is possible that some participants had unrecognised cognitive issues, given the high prevalence of MTA atrophy. However, it seems unlikely that many of the participants will have had frank dementia at study recruitment. Furthermore, there is no validated assessment of pre-stroke cognition ${ }^{25}$. Pre-stroke dementia may not be diagnosed till after presenting to stroke services and it could be argued that patients with MTA had AD prior to their index stroke event. This argument is not supported by our data, despite almost half of our included population exhibiting moderate to severe MTA, only six patients had a clinical diagnosis of dementia at baseline assessment. 
At one year, around a third of the study patients had MMSE $\leq 23$, suggestive of dementia. This finding suggests that pre-existing MTA may act as a marker of "brain frailty" pre-disposing a person to development of long term cognitive deficits. In this cognitively vulnerable state, incident stroke may be a sufficient cognitive "stressor" to lead to clinical cognitive impairment. MTA may therefore represent a useful in vivo biomarker to stratify stroke patients in clinical trials investigating cognitive outcomes. Such trials should include also MTA assessment as baseline radiological marker.

The strengths of our study are the standardized assessment of radiological variables with extensively validated radiological scales and the reproducibility of our methodology in clinical practice. The validated visual ratings we used are widely adopted, and cognitive function was screened using the MMSE, which is widely used by many stroke physicians. Although we recognize that MMSE is not a substitute of a comprehensive cognitive evaluation, in the context of clinical practice it is a useful and practical tool to detect those patients that require further cognitive assessment. Furthermore, it has been showed by a recent meta-analysis that MMSE is a reasonable tool to detect cognitive impairment among cognitive scales for evaluation of stroke patients ${ }^{18}$.

The main limitation of the study was the relatively small sample size. Our study may not have the statistical power to detect weaker but clinically meaningful associations with cognitive outcomes (e.g. SVD markers and cognition). However, the latest systematic review and meta-analysis of factors associated with post-stroke dementia examined about 600 patients with MTA assessment and found that MTA was associated with an increased risk of both pre- and post-stroke dementia ${ }^{25}$. Although comparable to previous similar studies ${ }^{20,21,24}$, we acknowledge that the sample size of the present was small. Nonetheless, our result considerably expands the dataset of the aforementioned meta-analysis, adding more than two hundred stroke patients with standardized MTA and SVD ratings. Moreover, the qualitative evaluation of MTA and SVD is easily transferrable to clinical practice.

We acknowledge that we did not included in the analysis important factors associated with post-stroke cognitive impairment, such as education level and stroke severity. As a retrospective study of existing datasets, we were constrained to variables evaluated in the original trials, and education level was not routinely assessed in the parent trials. Regarding stroke severity, we inserted in the analysis function level after stroke (i.e. disability) as a proxy of stroke severity. The use of a study population from clinical trials likely gave us a 
selected stroke population, however, the advantage of using trials dataset is in the quality of the collected data, which is often more complete than clinical registries.

Finally, we underscore that with cognitive data at a single time point we can only describe associations between factors of interest. A prospective cohort study could give more information on prognosis and possible directions of causation.

In conclusion, we have demonstrated that MTA, a radiological marker of $\mathrm{AD}$, is frequent among stroke survivors and is associated with radiological features of SVD, supporting a substantial overlap between vascular and degenerative pathology. Increasing severity of MTA is associated with short to medium term post-stroke cognitive impairment, and seems to have more weight than SVD features. Careful evaluation of radiological features pertaining to both $\mathrm{AD}$ and SVD in patients with stroke may help risk stratification for clinical practice and studies.

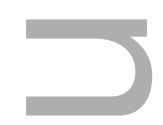

Acknowledgements: This study was partly supported by Tenovus Scotland. Dr Quinn is supported by a joint Stroke Association / Chief Scientist Office Senior clinical Lectureship.

Disclosures: the authors report no conflict of interest.

\section{*VISTA-Prevention Steering Committee:}

H-C. Diener (Chair), S. Davis, G. Hankey, K.R. Lees, B. Ovbiagele and C.J. Weir.

\section{References:}

1- Winblad B, Amouyel P, Andrieu S, et al. Defeating Alzheimer's disease and other dementias: a priority for European science and society. Lancet Neurol. 2016; 15: 455532.

2- $\quad$ Scheltens P, Leys D, Barkhof F, et al. Atrophy of medial temporal lobes on MRI in "probable" Alzheimer's disease and normal ageing: diagnostic value and neuropsychological correlates. J Neurol Neurosurg Psychiatry. 1992; 55:967-72.

3- Wardlaw JM, Smith C, Dichgans M. Mechanisms of sporadic cerebral small vessel disease: insights from neuroimaging. Lancet Neurol. 2013; 12:483-97.

4- Gorelick PB, Scuteri A, Black SE, et al. ; American Heart Association Stroke Council, Council on Epidemiology and Prevention, Council on 
Cardiovascular Nursing, Council on Cardiovascular Radiology and Intervention, and Council on Cardiovascular Surgery and Anesthesia. Vascular contributions to cognitive impairment and dementia: a statement for healthcare professionals from the american heart association/american stroke association. Stroke. 2011; 42:2672-713.

5- Kuller LH, Lopez OL, Newman A, et al. Risk factors for dementia in the cardiovascular health cognition study. Neuroepidemiology. 2003; 22:13-22.

6- Launer LJ, Ross GW, Petrovitch H, Masaki K, Foley D, White LR, Havlik RJ. Midlife blood pressure and dementia: the Honolulu-Asia aging study. Neurobiol Aging. 2000; 21:49-55.

7- Ott A, Stolk RP, van Harskamp F, Pols HA, Hofman A, Breteler MM. Diabetes mellitus and the risk of dementia: The Rotterdam Study. Neurology. 1999; 53:193742.

8- $\quad$ Leys D, Hénon H, Mackowiak-Cordoliani MA, Pasquier F. Poststroke dementia. Lancet Neurol. 2005; 4: 752-9.

9- Brainin M, Tuomilehto J, Heiss WD, et al.; Post Stroke Cognition Study Group. Poststroke cognitive decline: an update and perspectives for clinical research. Eur J Neurol. 2015; 22:229-38, e13-6.

10- Ali M, Bath P, Brady M, et al.; VISTA Steering Committees. Development, expansion, and use of a stroke clinical trials resource for novel exploratory analyses. Int J Stroke. 2012; 7:133-8.

11- Adams HP Jr, Bendixen BH, Kappelle LJ, et al. Classification of subtype of acute ischaemic stroke. Definitions for use in a multicenter clinical trial. TOAST. Trial of Org 10172 in Acute Stroke Treatment. Stroke. 1993; 24:35-41.

12- Bamford JM, Sandercock P, Warlow C, et al. Interobserver Agreement for the Assessment of Handicap in Stroke Patients. Stroke. 1989; 20:828-828.

13- Wardlaw JM, Smith EE, Biessels GJ, et al.; STandards for ReportIng Vascular changes on nEuroimaging (STRIVE v1). Neuroimaging standards for research into small vessel disease and its contribution to ageing and neurodegeneration. Lancet Neurol. 2013; 12:822-38. 
14- Van Swieten JC, Hijdra A, Koudstaal PJ, van Gijn J. Grading white matter lesions on CT and MRI: a simple scale. J Neurol Neurosurg Psychiatry. 1990; 53:1080-3.

15- IST-3 collaborative group. Association between brain imaging signs, early and late outcomes, and response to intravenous alteplase after acute ischaemic stroke in the third International Stroke Trial (IST-3): secondary analysis of a randomised controlled trial. Lancet Neurol. 2015; 14:485-96.

16- Potter GM, Chappell FM, Morris Z, et al. Cerebral perivascular spaces visible on magnetic resonance imaging: development of a qualitative rating scale and its observer reliability. Cerebrovasc Dis. 2015; 39:224-31.

17- Folstein MF, Folstein SE, McHugh PR. "Mini-mental state". A practical method for grading the cognitive state of patients for the clinician. J Psychiatr Res. 1975;12:18998.

18- Lees R, Selvarajah J, Fenton C, et al. Test accuracy of cognitive screening tests for diagnosis of dementia and multidomain cognitive impairment in stroke. Stroke. 2014; 45:3008-18.

19- Hénon H, Pasquier F, Durieu I, Pruvo JP, Leys D. Medial temporal lobe atrophy in stroke patients: relation to pre-existing dementia. J Neurol Neurosurg Psychiatry. 1998; 65: 641-7.

20- Kebets V, Gregoire SM, Charidimou A, et al. . Prevalence and cognitive impact of medial temporal atrophy in a hospital stroke service: retrospective cohort study. Int J Stroke. 2015 ; 10: 861-7.

21- Jokinen H, Kalska H, Ylikoski R, et al. . Medial temporal lobe atrophy and memory deficits in elderly stroke patients. Eur J Neurol. 2004; 11:825-32.

22- Scheltens P, Barkhof F, Valk J, et al. White matter lesions on magnetic resonance imaging in clinically diagnosed Alzheimer's disease. Evidence for heterogeneity. Brain. 1992; 115 ( Pt 3):735-48.

23- Bastos-Leite AJ, van der Flier WM, van Straaten EC, Staekenborg SS, Scheltens P, Barkhof F. The contribution of medial temporal lobe atrophy and vascular pathology to cognitive impairment in vascular dementia. Stroke. 2007; 38:3182-5. 
24- Firbank MJ, Burton EJ, Barber R, et al. Medial temporal atrophy rather than white matter hyperintensities predict cognitive decline in stroke survivors. Neurobiol Aging. 2007; 28:1664-9.

25- McGovern A, Pendlebury ST, Mishra NK, Fan Y, Quinn TJ. Test Accuracy of Informant-Based Cognitive Screening Tests for Diagnosis of Dementia and Multidomain Cognitive Impairment in Stroke. Stroke. 2016; 47:329-35.

26- Pendelbury ST, Rothwell PM. Prevalence, incidence, and factors associated with prestroke and post-stroke dementia: a systematic review and meta-analysis. Lancet Neurol 2009; 8:1006-18.

Table 1. Baseline demographic, clinical and radiological characteristics of study population.

\begin{tabular}{|l|c|c|c|c|}
\hline Variables & $\begin{array}{c}\text { All } \\
\text { N=234 }\end{array}$ & $\begin{array}{c}\text { MTA 0-1 } \\
\text { N=130 }\end{array}$ & $\begin{array}{c}\text { MTA 2-4 } \\
\text { N=104 }\end{array}$ & p \\
\hline Age, mean ( \pm SD) & $65.7 \pm 13.1$ & $60.2( \pm 13.3)$ & 72.5 & $<0.001$ \\
$( \pm 9.0)$ & \\
\hline Sex (male) & $145(62)$ & $78(60)$ & $67(64)$ & 0.489 \\
\hline Ischaemic stroke & $195(83)$ & $105(81)$ & $90(87)$ & 0.297 \\
\hline Lacunar stroke & $94(40)$ & $46(43.8)$ & $48(53.3)$ & 0.185 \\
\hline Hypertension & $185(79)$ & $95(73)$ & $90(87)$ & 0.012 \\
\hline Diabetes & $70(30)$ & $33(26)$ & $37(36)$ & 0.098 \\
\hline Hypercholesterolemia & $129(55)$ & $73(61)$ & $56(57)$ & 0.475 \\
\hline Atrial fibrillation & $13(6)$ & $7(5)$ & $6(6)$ & 0.898 \\
\hline Ischaemic heart disease & $35(15)$ & $19(15)$ & $16(15)$ & 0.870 \\
\hline Peripheral vascular disease & $3(1)$ & $3(2)$ & - & 0.119 \\
\hline Smoker & $47(20)$ & $24(19)$ & $23(22)$ & 0.465 \\
\hline Depression & $15(6)$ & $10(8)$ & $5(5)$ & 0.363 \\
\hline OHS, median (IQR) & $1(1-2)$ & $1(1-2)$ & $2(1-3)$ & $<0.001$ \\
\hline Van Swieten Scale $>2$ & $83(36)$ & $21(16)$ & $62(60)$ & $<0.001$ \\
\hline Lacunes $\geq 2$ & $137(60)$ & $62(48)$ & $75(72)$ & $<0.001$ \\
\hline EPVS Basal ganglia $\geq 2$ & $121(52)$ & $45(35)$ & $76(73)$ & $<0.001$ \\
\hline EPVS centrum semiovale $\geq 2$ & $76(59)$ & $85(82)$ & $<0.001$ \\
\hline
\end{tabular}




\begin{tabular}{|l|l|l|l|l|}
\hline Brain atrophy central >2 & $60(25)$ & $8(6)$ & $52(50)$ & $<0.001$ \\
\hline Brain atrophy cortical >2 & $42(18)$ & $6(5)$ & $36(35)$ & $<0.001$ \\
\hline
\end{tabular}

Statistical tests: age (ANOVA), OHS (Mann-Whitney U test), all the other variables $\left(\chi^{2}\right.$ test).

MTA= Medial Temporal lobe Atrophy; SD=Standard Deviation; OHS=Oxford Handicap Scale; IQR=Interquartile Range; EPVS=Enlarged Perivascular Spaces.

Table 2. Spearman correlation between MTA and small vessel disease markers.

\begin{tabular}{|l|l|c|}
\cline { 2 - 3 } & Variables & Any MTA \\
\cline { 2 - 3 } & Van Swieten Scale & 0.60 \\
\cline { 2 - 3 } & Lacunes & 0.32 \\
\cline { 2 - 3 } & Basal Ganglia EPVS & 0.44 \\
\cline { 2 - 3 } & Centrum Semiovale EPVS & 0.35 \\
\cline { 2 - 3 } & Central volume reduction & 0.64 \\
\hline & Cortical Atrophy & 0.49 \\
\hline
\end{tabular}

All $\mathrm{p}$ values are $<0.001$.

Table 3. Logistic regression showing associations between radiological markers of small vessel disease and medial temporal lobe atrophy dichotomized (0-1 vs 2-4).

\begin{tabular}{|l|c|}
\hline Variable & MTA 2-4 \\
\hline & OR $(\mathbf{9 5 \%}$ CI $)$ \\
\hline Van Swieten Scale & $2.74(1.95-3.85)$ \\
\hline Lacunes & $1.44(1.15-1.81)$ \\
\hline Basal ganglia EPVS & $1.99(1.31-3.02)$ \\
\hline Centrum Semiovale EPVS & $1.47(0.99-2.18)$ \\
\hline
\end{tabular}




\begin{tabular}{|l|l|}
\hline Central brain atrophy & 5.87 (3.14-10.97) \\
\hline Cortical brain atrophy & $4.54(2.14-9.63)$ \\
\hline
\end{tabular}

MTA=Medial Temporal lobe Atrophy; OR=Odds Ratio; CI=Confidence Interval;

EPVS=Enlarged perivascular spaces.

We ran separate logistic regressions for each row adjusting for: age, sex, hypertension, diabetes.

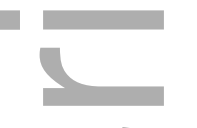

Table 4. Multivariate associations between radiological features and cognitive status one year after stroke.

\begin{tabular}{|c|c|c|c|c|}
\hline ᄀ & \multicolumn{4}{|c|}{ OR $(95 \%$ CI $)$} \\
\hline Variables & $\begin{array}{c}\text { MMSE } \leq 26 \\
\text { (Unadjusted) } \\
\text { N=101 }\end{array}$ & $\begin{array}{c}\text { MMSE } \leq 26 \\
\text { (Adjusted) } \\
\text { N=101 }\end{array}$ & $\begin{array}{c}\text { MMSE } \leq 23 \\
\text { (Unadjusted) } \\
\text { N=67 }\end{array}$ & $\begin{array}{c}\text { MMSE } \leq 23 \\
\text { (Adjusted) } \\
\mathrm{N}=67\end{array}$ \\
\hline $\begin{array}{l}\text { Temporal lobe atrophy } \\
\text { (MTA) }\end{array}$ & $\begin{array}{c}2.87(2.04-4- \\
04)\end{array}$ & $1.94(1.28-2.94)$ & $\begin{array}{c}3.07(2.15- \\
4.38)\end{array}$ & $\begin{array}{c}2.31(1.48- \\
3.62)\end{array}$ \\
\hline White matter changes & $\begin{array}{c}1.41(1.08- \\
2.30)\end{array}$ & $1.01(0.73-1.38)$ & $\begin{array}{c}1.86(1.44- \\
2.41)\end{array}$ & $\begin{array}{c}1.12(0.79- \\
1.58)\end{array}$ \\
\hline Number of lacunes & $\begin{array}{c}1.26(1.02- \\
1.57)\end{array}$ & $1.07(0.82-1.39)$ & $\begin{array}{c}1.20(0.97- \\
1.49)\end{array}$ & * \\
\hline Basal ganglia EPVS & $\begin{array}{l}2.81(1.93- \\
4.11)\end{array}$ & $1.37(0.87-2.17)$ & $\begin{array}{c}2.66(1.82- \\
3.87)\end{array}$ & $\begin{array}{c}1.43(0.89- \\
2.29)\end{array}$ \\
\hline $\begin{array}{l}\text { Centrum Semiovale } \\
\text { EPVS }\end{array}$ & $\begin{array}{c}1.79(1.28- \\
2.51)\end{array}$ & $0.95(0.61-1.48)$ & $\begin{array}{c}2.08(1.42-3- \\
05)\end{array}$ & $\begin{array}{c}1.16(0.71- \\
1.88)\end{array}$ \\
\hline Central brain atrophy & $\begin{array}{c}2.33(1.52- \\
3.58)\end{array}$ & $1.20(0.68-2.13)$ & $\begin{array}{c}2.28(1.45- \\
3.58)\end{array}$ & $\begin{array}{c}1.26(0.69- \\
2.32)\end{array}$ \\
\hline Cortical brain atrophy & $\begin{array}{c}4.39(2.40- \\
8.02)\end{array}$ & $2.02(0.96-4.23)$ & $\begin{array}{c}3.25(1.83- \\
5.77)\end{array}$ & $\begin{array}{c}1.53(0.69- \\
3.37)\end{array}$ \\
\hline
\end{tabular}

*Analysis not done due to lack of significance at the univariate analysis.

MMSE=Mini Mental State Examination; OR=Odds ratio; $\mathrm{CI}=$ Confidence Interval EPVS=Enlarged Perivascular Spaces. 
We ran separate logistic regressions for each row adjusting for: age, sex, OHS baseline, hypertension, diabetes.

Figure 1. Distribution of one year MMSE across medial temporal lobe atrophy scores.

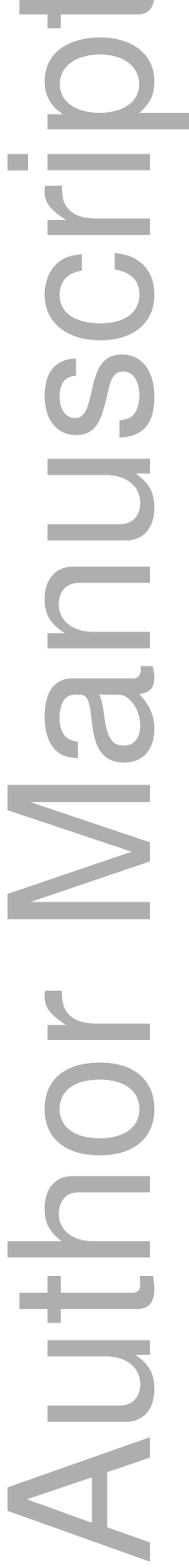


Figure 1. Distribution of one year MMSE across medial temporal lobe atrophy scores.

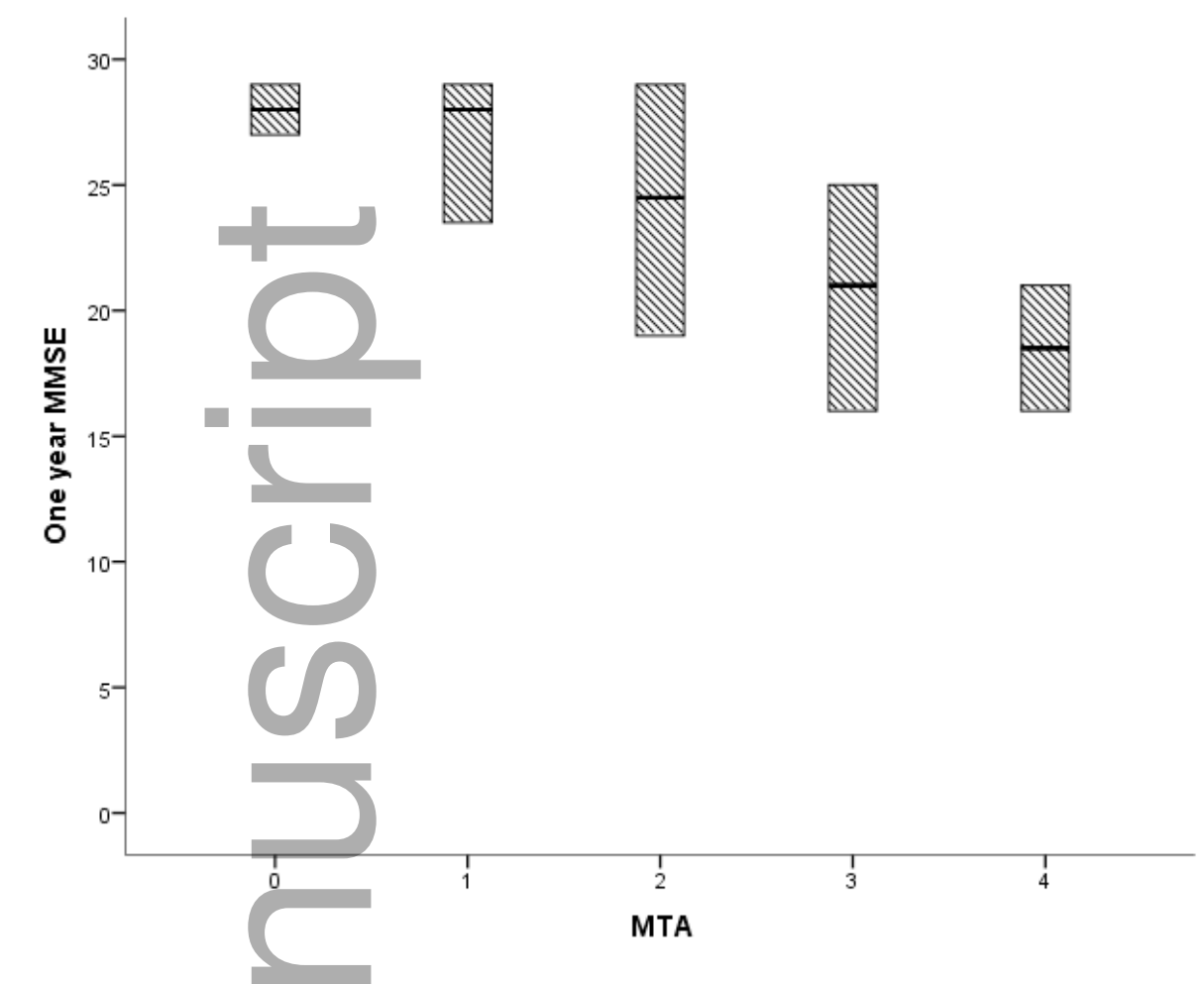

$\mathrm{p}<0.001$ (Kruskal-Wallis)
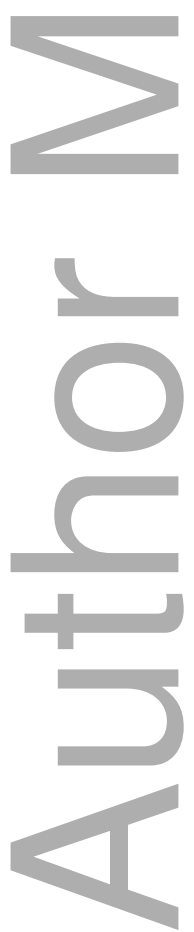

This article is protected by copyright. All rights reserved 


\section{University Library}

\section{- M M N E R VA A gateway to Melbourne's research publications}

Minerva Access is the Institutional Repository of The University of Melbourne

Author/s:

Arba, F;Quinn, T;Hankey, GJ;Ali, M;Lees, KR;Inzitari, D

Title:

Cerebral small vessel disease, medial temporal lobe atrophy and cognitive status in patients with ischaemic stroke and transient ischaemic attack

Date:

2017-02-01

Citation:

Arba, F., Quinn, T., Hankey, G. J., Ali, M., Lees, K. R. \& Inzitari, D. (2017). Cerebral small vessel disease, medial temporal lobe atrophy and cognitive status in patients with ischaemic stroke and transient ischaemic attack. EUROPEAN JOURNAL OF NEUROLOGY, 24 (2), pp.276-282. https://doi.org/10.1111/ene.13191.

Persistent Link:

http://hdl.handle.net/11343/292129 